\title{
Photonic Hall Effect in ferrofluids: Theory and Experiments
}

\author{
D. Lacoste ${ }^{a}$ f, F. Donatini ${ }^{b}$, S. Neveu ${ }^{c}$, J. A. Serughetti ${ }^{d}$ and B. A. Van Tiggelen ${ }^{e}$ \\ ${ }^{a}$ Department of Physics, \\ University of Pennsylvania, Philadelphia, PA 19104, USA \\ ${ }^{b}$ LRCCI, Université Jean-Monnet, \\ 23 rue Docteur P. Michelon, 42023 St-Etienne Cedex 2, France \\ ${ }^{c}$ LI2C, 4 place Jussieu, Case courrier 63, 75252 PARIS, France \\ ${ }^{d}$ Université Claude Bernard Lyon I, \\ 43 Blvd du 11 novembre 1918, 69622 Villeurbanne CEDEX, France \\ ${ }^{e}$ LPM2C, Maison des Magistères Jean-Perrin, \\ CNRS-BP. 166, 38042 Grenoble Cedex 9, France
}

\begin{abstract}
An experimental and theoretical study on the Photonic Hall Effect (PHE) in liquid and gelled samples of ferrofluids is presented. The ferrofluids are aqueous colloidal suspensions of $\mathrm{Fe}_{2} \mathrm{CoO}_{4}$ particles, which can be considered as anisotropic and absorbing Rayleigh scatterers. The PHE is found to be produced by the orientation of the magnetic moments of the particles, as is also the case for the Faraday effect. The dependence of the PHE with respect to the concentration of the scatterers, the magnetic field and the polarization of the incident light is measured in liquid and in gelled samples and is compared to a simple model based on the use of a scattering matrix and the single scattering approximation.
\end{abstract}

PACS numbers: 78.20.L, 83.80.G, 78.35

*e-mail: lacoste@dept.physics.upenn.edu 


\section{INTRODUCTION}

Magneto-transverse light diffusion - more popularly known as the "Photonic Hall Effect" (PHE) - was theoretically predicted five years ago by Van Tiggelen [1], and was experimentally confirmed one year later by Rikken [2]. This effect is analogous to the well-known electronic Hall effect, although the origin of the PHE is somewhat different: The PHE finds its origin in the Faraday effect, present inside the dielectric scatterers, which slightly changes their scattering amplitude. Most experiments on the PHE reported so far used solid samples comprising paramagnetic or diamagnetic particles, of size much larger than the wavelength (the Mie scattering regime) 2]. The scatterers were embedded in a medium having no magnetic properties, with a volume fraction such that multiple light scattering prevailed. A perturbational formulation of the Mie scattering of a Faraday-active sphere has been developed to explain the origin of the PHE in single scattering [3]. A transport theory for light based on this formulation, could produce an estimate of the PHE in multiple light scattering, which agreed with experiments 4 . Experiments have checked the validity of this formulation by measuring the PHE as a function of the volume fraction of the scatterers, of the wavelength or of the index of refraction even in the presence of absorption [5].

These experiments can be difficult due to the smallness of the measured PHE. It seems therefore natural to try to use other samples such as samples containing ferromagnetic particles, in which the magneto-optical effects are expected to be much larger than in paramagnetic or diamagnetic samples. The giant magneto-optical properties of ferromagnetic compounds were explained by a microscopic theory due to Argyres, who derived the form of their polarizability and conductivity tensors 6]. This approach was confirmed by Krinchik, whose classical model was able to explain both the Faraday effect at optical frequency and the ferromagnetic Hall effect, which is the classical Hall effect for the DC magneto-transverse electrical conductivity [7].

This paper deals with the PHE of liquid or gelled samples of ferrofluids, which are colloidal suspensions of ferromagnetic particles. The experiments reported here are also the first experimental realization of the PHE in a medium comprising Rayleigh scatterers. In these samples, the significant absorption precludes the observation of multiple light scattering which does not exclude the PHE. A sketch of the geometry is shown in Fig. 1. This geometry is called magneto-transverse (or Voigt geometry) since the magnetic field and the incident light are perpendicular. These two perpendicular directions define a plane, and the total scattered intensity above this plane is called $I^{+}$and the one below this plane it is called $I^{-}$. The PHE is a manifestation of an anisotropy in the scattered light, which produces a net difference between $I^{+}$and $I^{-}$, linear in the magnetic field. The quantity $\eta$, which is a measure of the PHE, is defined as the difference between these intensities $\Delta I(\mathbf{B})=I^{+}(\mathbf{B})-I^{-}(\mathbf{B})$ normalized with respect to the average of the scattered intensities in the absence of any magnetic field denoted $I_{0}$,

$$
\eta=\frac{2\left(I^{+}(\mathbf{B})-I^{-}(\mathbf{B})\right)}{I^{+}(\mathbf{B}=0)+I^{-}(\mathbf{B}=0)}=\frac{\Delta I(\mathbf{B})}{I_{0}} .
$$

The difference $\Delta I(\mathbf{B})$ is the photonic equivalent of the difference of potential in the electronic Hall effect. In dilute ferrofluids, the ratio $\eta$ can be as high as $10^{-3}$ for a magnetic field of $100 \mathrm{Oe}$, which is two or three orders of magnitude above the value measured in paramagnetic samples under the same conditions [2].

This paper is organized as follows: Section $\amalg$ recalls some general properties of ferrofluids, section III deals with their magneto-optical properties, section IV presents a theoretical model for the PHE, and section V contains the experimental study of the PHE in liquid and gelled samples of ferrofluids. 


\section{FERROFLUIDS}

The stability of magnetic liquids can be destroyed by several factors such as gravity, interaction between magnetic moments, gradients in the applied magnetic field, and Van der Waals interactions, which favor sedimentation or agglomeration. In practice, the stability of the suspension is achieved by chemically grafting charged substituents or polymers on the particles, in order to increase the repulsion between the particles, of ionic origin (in aqueous ferrofluids), or of stearic origin (in organic ferrofluids). Because of these stability requirements, the size of the particles in the suspension is limited to a radius of a few nanometers 8]. The ferrofluids used in all experiments discussed in this paper, were synthesized by Neveu in the Laboratoire de PhysicoChimie Inorganique in Jussieu using a coprecipitation technique first devised by Prof. Massart (1981). The samples are suspensions of ferrite particles $\mathrm{Fe}_{2} \mathrm{CoO}_{4}$ dispersed in an aqueous ionic solution of citrate.

In such a suspension, each particle is a magnetic single-domain, of magnetic moment

$$
\mu=4 M_{s} \mu_{0} \pi a^{3} / 3 \approx 4,1 \cdot 10^{4} \mu_{B},
$$

where $M_{s} \simeq 422 \mathrm{kAm}^{-1}$ is the saturation magnetization of the bulk material, and $\mu_{B}$ is Bohr's magneton. From this average magnetic moment and the average distance between particles $r$, a dipolar interaction energy $E_{d}=\left(\mu_{0} / 4 \pi\right) \mu^{2} / r^{3}$ can be estimated. For the most concentrated of our samples of volume fraction $f=1 \%$, the magnetic interactions are still negligible since at room temperature $E_{d} / k_{B} T \simeq 0.17$. With such a small value, there should be essentially no chain structures in the magnetic liquid. The formation of chain structures in magnetic liquids was predicted long ago by De Gennes and Pincus [9], and was studied more recently by Stevens and Grest using numerical simulations [10].

In the absence of any magnetic field, the magnetic moments $\mu$ have random orientation and the net average magnetization of the fluid is zero. When a magnetic field is applied, there is a tendency for the particles to align in the direction of the magnetic field. Langevin's theory of paramagnetic gases applies to ferrofluids provided that the magnetic interaction are negligible. The behavior of these magnetic fluids is known as superparamagnetism 88. The statistical average of the magnetic moments $\bar{\mu}$ is oriented along the magnetic field and its amplitude is

$$
\bar{\mu}=\mu L(u),
$$

where $u=\mu B / k_{B} T$ for an applied magnetic field $B$ and $L(u)$ is the Langevin function. It is recalled that $L(u)=\operatorname{coth} u-1 / u$, and $L(u) \simeq u / 3$ for $u \ll 1$. Quantitative comparisons with experiments have shown that a polydispersity function should be added to Eq. (3). Particles sizes obey a log-normal distribution characterized by an average radius $a$ and a variance.

The relaxation of the magnetization is known to have two possible mechanisms: either it is caused by a rotation of the particles within the liquid, or by the rotation of the magnetic moments inside the particles with respect to their easy magnetization axis. The first mechanism is characterized by the Brownian time relaxation 8

$$
\tau_{B}=\frac{3 V^{\prime} \nu}{k_{B} T}
$$

where $V^{\prime}$ is the hydrodynamic volume of the particle, and $\nu$ is the viscosity of the carrier liquid. The second mechanism is characterized by the Néel relaxation time

$$
\tau_{N}=\frac{1}{f_{0}} \exp \left(\frac{K V}{k_{B} T}\right)
$$


where $K$ is the anisotropy constant, $V$ the particle volume, and $f_{0}$ is a characteristic frequency of the order of $10^{9} \mathrm{~Hz}$. For Cobalt ferrofluids and for a magnetic field of frequency $\omega_{H}$, we have $\tau_{B} \ll \omega_{H}^{-1} \ll \tau_{N}$. Therefore Néel relaxation is negligible in these samples and the dynamic behavior of the fluids follows Brownian relaxation. The magnetic moments of the particles in Cobalt ferrite samples remain fixed with respect to the particles as confirmed by studies of the linear birefringence in these compounds 11.

\section{MAGNETO-OPTICS OF FERROFLUIDS}

\section{A. Optical properties in single scattering}

Some aspects of the optical properties of ferrite Cobalt samples are now briefly discussed. The relative dielectric constant $\kappa_{0}$ in the absence of magnetic field is obtained from the dielectric constant of the bulk material, which equals $2.6-0.7 i$ at $\lambda_{0}=477 \mathrm{~nm}$ according to Refs. 12,13. The average radius of the particles is estimated to be $a=6 \mathrm{~nm}$. This corresponds to Rayleigh scattering, since the average size parameter, $x=2 \pi a n_{\text {water }} / \lambda_{0} \simeq 0.1$, which is the ratio of the size of the particle to the wavelength in the medium is smaller than one as is also the case for the other size parameter $y=\sqrt{\kappa_{0}^{\prime}} x n_{\text {water }} \simeq 0.2$. For these Rayleigh absorbing particles, extinction is dominated by absorption because of the behavior of the scattering and absorption cross-section as function of $x$ 14. For $x \ll 1$, and when the imaginary part of the index of the scatterer is smaller than its real part (which is the case with these ferrofluids), the following relations hold

$$
Q_{a b s}=4 x \operatorname{Im}\left(\frac{\kappa_{0}-1}{\kappa_{0}+2}\right) \gg \mathrm{Q}_{\mathrm{scatt}}=\frac{8 \mathrm{x}^{4}}{3}\left|\frac{\kappa_{0}-1}{\kappa_{0}+2}\right|^{2} .
$$

The imaginary part of the index can therefore be determined from the absorption spectra provided that the real part of the index is also known. Figure (2) represents the absorption length as function of the volume fraction on a logarithmic scale. The measurements were taken with a spectrometer, and the length of the sample was changed so that the absorbance, which is defined as the logarithm of the transmission, be of order unity. The agreement with the linear behavior predicted from Lambert's law, with the slope computed from Rayleigh scattering theory, shows that our estimates concerning the index of refraction and the size of the particles (assuming a monodisperse suspension) are basically correct. The generalization to the case of polydisperse samples did not appear to be necessary for this estimate.

\section{B. Transport properties}

Typical values of the absorption length $\ell_{a b s}$ of the coherent beam and of the transport mean free path $\ell^{*}$ in a sample of volume fraction $f=0.14 \%$, at the wavelength $\lambda_{0}=477 \mathrm{~nm}$, are $\ell_{a b s}=80 \mu \mathrm{m}$ (measured) and $\ell^{*}=6 \mathrm{~cm}$ (theoretically estimated). For these samples made of particles smaller than the wavelength, the transport mean free path $\ell^{*}$ is very close to the elastic mean free path $\ell$. A treatment of multiple light scattering with absorption, becomes necessary when the elastic mean free path is smaller than the sample size. For all the samples discussed in this paper, the volume fraction is smaller than $f=0.1 \%$, and the condition $\ell \gg L$ is fulfilled at this wavelength, which means that multiple scattering should be negligible.

Because of the importance of absorption, single scattering should therefore prevail. It does not mean however that interference effects are absent, since scattered waves 
may still interfere as long as they have traveled a distance shorter or comparable to the absorption length of the coherent beam $\ell_{a b s}$. This idea will be employed in the model of section IV.

\section{Magneto-optical properties}

In the absence of an applied magnetic field, ferrofluids behave as a normal liquid, and no birefringence or dichroism, circular or linear, are expected to be present. The application of a magnetic field introduces magneto-optical anisotropy, which has been the subject of many experimental studies 15.16. In the longitudinal configuration, in which the wave vector of the incident light is directed along the applied magnetic field, the eigenmodes of the electric field are circularly polarized waves. The difference in speed and absorption of these waves results in circular birefringence and dichroism, which is related to Faraday rotation and ellipticity. The Faraday rotation and ellipticity are odd functions of the applied field and are linear at small magnetic field as shown in Fig. 3. In this figure, only one curve is plotted, since the curves of the Faraday rotation and ellipticity are both proportional to the same Langevin function. This was confirmed experimentally by measuring the sample magnetization, the Faraday rotation and ellipticity as a function of the magnetic field at the LRCCI laboratory with samples of $\mathrm{Fe}_{3} \mathrm{O}_{4}$. All curves superimpose until volume fraction of a several percents 17. This experiment confirms that the Faraday rotation and ellipticity in ferrofluids have the same physical origin, which is the orientation of the magnetic moments in the direction of the magnetic field.

In the magneto-transverse or Voigt configuration, the eigenmodes of the electric field are linearly polarized waves. This implies linear birefringence and dichroism, which are both even functions of the magnetic field. Below a critical field of about 50 Oe for the Cobalt ferrite, these effects are quadratic in the applied magnetic field. Above this critical field, the effects are linear but still of course even functions of the applied magnetic field 18. The linear birefringence and dichroism are also explained by Langevin theory 19], as shown by the pioneering experiments of Bacri et al. [11,20]. The standard Langevin model however, fails to describe the birefringence in spatially ordered samples or in samples in which the particles are not completely free to move. This can be the case for instance if the particles are gelled or binded to some substrate. The magneto-optical properties of gels were studied theoretically and experimentally in magnetic layered silica gels in Refs. 21,22. In random solgels of the type considered in this paper, the four magneto-optical effects have been measured 23]. These experiments have shown that the linear magneto-optical effects can be inhibited in sol-gels, whereas the circular magneto-optical effects are always present and essentially do not change in the gels as compared to the liquids.

\section{MODEL FOR THE PHE IN FERROFLUIDS}

\section{A. Single scattering $\mathbf{T}$ matrix}

A simple model is presented to describe the Photonic Hall Effect in liquid and gelled samples of ferrofluids. The samples are dilute and the predominant scattering corresponds to single scattering, which is described using a $\mathrm{T}$ matrix formulation.

Our notations are as follows: $\hat{\mathbf{k}}$ and $\hat{\mathbf{k}}^{\prime}$ are the wave vectors of the incoming and outgoing light respectively and $\hat{\mathbf{B}}$ is the direction of the applied field. With each of these three vectors, it is possible to associate a local right-handed coordinate system, having its $z$-axis along the wave vector or along the magnetic field. The $x$ and $y$ 
axis are chosen such that $\hat{\mathbf{B}}$ and $\hat{\mathbf{k}}$ are perpendicular, as is the case in the magnetotransverse geometry.

The incoming beam can be regarded as a linear superposition of plane waves, each having a transverse electric field

$$
\mathcal{E}_{i n c}=\mathbf{E}_{i n c} \exp [i(k z-\omega t+\delta)]
$$

with

$$
\mathbf{E}_{\text {inc }}=E_{1} \hat{\mathbf{g}}_{1}+E_{2} \hat{\mathbf{g}}_{2} .
$$

The wave vector of the light in the medium is denoted $k$, and the phase $\delta$ can be taken as a constant. A convenient choice of polarization vectors is: $\hat{\mathbf{g}}_{1}=\hat{\mathbf{g}}=\hat{\mathbf{k}} \times \hat{\mathbf{k}}^{\prime} /\left|\hat{\mathbf{k}} \times \hat{\mathbf{k}}^{\prime}\right|$, $\hat{\mathbf{g}}_{2}=\hat{\mathbf{k}} \times \hat{\mathbf{g}}_{1}$. The scattered beam can be decomposed in the polarization vectors $\hat{\mathbf{g}}_{2}^{\prime}=\hat{\mathbf{k}}^{\prime} \times \hat{\mathbf{g}}_{1}$ and $\hat{\mathbf{g}}_{1}^{\prime}=\hat{\mathbf{g}}_{1}$. This choice of polarization vectors was introduced by Van De Hulst: one polarization vector is located in the scattering plane while the other one is perpendicular to it 24]. This choice is not well defined in the particular cases of forward and backward scattering because there is no scattering plane in these cases, but these two particular configurations are not relevant for the PHE. In the frame of the particle, in which quantities are denoted with a prime, the scattering matrix reads

$$
\mathbf{t}^{\prime}=\left(\begin{array}{ccc}
t_{0}^{\prime} & i t_{1}^{\prime} & 0 \\
-i t_{1}^{\prime} & t_{0}^{\prime} & 0 \\
0 & 0 & t_{0}^{\prime}+t_{2}^{\prime}
\end{array}\right)
$$

The complex-valued coefficients $t_{0}^{\prime}, t_{1}^{\prime}$ and $t_{2}^{\prime}$ describe a point-like scatterer in a magnetic field.

To show the relationship between the properties of a single particle and the macroscopic magneto-optical properties of an ensemble of particles, a statistical average of the magnetic moments of the particles has to be performed, as discussed in Ref. [26. When the coupling between the magnetic moments is neglected, and when a random distribution of scatterers is assumed, the average $\mathrm{T}$ matrix has the same form as Eq. (G) with the following parameters $t_{0}=t_{0}^{\prime}+t_{2}^{\prime} L(u) / u, t_{1}=t_{1}^{\prime} L(u)$ and $t_{2}=t_{2}^{\prime} L_{2}(u)=t_{2}^{\prime}(1-3 L(u) / u)$, where $u=\mu B / k_{B} T$ for an applied magnetic field $B$, since

$$
\mathbf{t}=\overline{\mathbf{t}^{\prime}}=\left(\begin{array}{ccc}
t_{0}^{\prime}+t_{2}^{\prime} L(u) / u & i t_{1}^{\prime} L(u) & 0 \\
-i t_{1} L(u) & t_{0}^{\prime}+t_{2}^{\prime} L(u) / u & 0 \\
0 & 0 & t_{0}^{\prime}+t_{2}^{\prime}(1-2 L(u) / u)
\end{array}\right)
$$

This result implies that the Faraday rotation associated with $t_{1}$, is proportional to the Langevin function $L(u)$, and that the linear birefringence is associated with the second Langevin function $L_{2}(u)$ contained in $t_{2}$, as confirmed by experiments.

The scattering matrix, which relates linearly the outgoing and the incoming electric field needs now to be expressed in the basis of the polarization vectors introduced above. This yields to the following result:

$$
\begin{gathered}
S_{11}=t_{0}+t_{2}(\hat{\mathbf{B}} \cdot \hat{\mathbf{g}})^{2}, \\
S_{12}=-t_{2} \frac{\left(\hat{\mathbf{B}} \cdot \hat{\mathbf{k}}^{\prime}\right)(\hat{\mathbf{B}} \cdot \hat{\mathbf{g}})}{\left|\hat{\mathbf{k}} \times \hat{\mathbf{k}}^{\prime}\right|}, \\
S_{21}=-i t_{1} \hat{\mathbf{B}} \cdot \hat{\mathbf{k}}^{\prime}-t_{2} \frac{\left(\hat{\mathbf{B}} \cdot \hat{\mathbf{k}}^{\prime}\right)(\hat{\mathbf{B}} \cdot \hat{\mathbf{g}})\left(\hat{\mathbf{k}} \cdot \hat{\mathbf{k}}^{\prime}\right)}{\left|\hat{\mathbf{k}} \times \hat{\mathbf{k}}^{\prime}\right|},
\end{gathered}
$$




$$
S_{22}=t_{0} \hat{\mathbf{k}} \cdot \hat{\mathbf{k}}^{\prime}+t_{2} \frac{\left(\hat{\mathbf{k}} \cdot \hat{\mathbf{k}}^{\prime}\right)\left(\hat{\mathbf{B}} \cdot \hat{\mathbf{k}}^{\prime}\right)^{2}}{\left|\hat{\mathbf{k}} \times \hat{\mathbf{k}}^{\prime}\right|^{2}}-i t_{1} \hat{\mathbf{B}} \cdot\left(\hat{\mathbf{k}} \times \hat{\mathbf{k}}^{\prime}\right) .
$$

These expressions are valid only for the magneto-transverse geometry but at any order in the magnetic field. They can be simplified by introducing a coordinate system in which the wave vector of the scattered wave $\hat{\mathbf{k}}^{\prime}$ makes an angle $\theta$ in the scattering plane with respect to the incoming direction and an azimuthal angle $\varphi$ with respect to $\hat{\mathbf{B}}$ as shown in figure 1 . With this choice,

$$
\hat{\mathbf{B}}=\left(\begin{array}{l}
1 \\
0 \\
0
\end{array}\right), \quad \hat{\mathbf{k}}=\left(\begin{array}{l}
0 \\
0 \\
1
\end{array}\right), \quad \hat{\mathbf{k}}^{\prime}=\left(\begin{array}{c}
\sin \theta \cos \varphi \\
\sin \theta \sin \varphi \\
\cos \theta
\end{array}\right)
$$

\section{B. PHE for unpolarized incident light}

The scattering matrix of Eqs. $8 \mathrm{a}$ ( $)$ describes single scattering. This approach is not sufficient to account for the PHE in ferrofluids, because the PHE of a single magnetic Rayleigh scatterer is zero as was found in Ref. 3. It is therefore necessary to take into account interferences among different single scattering events, in order to explain the observed non-zero value of the PHE. To this end, one can start with the scattering matrix of a set of particles at position $\mathbf{r}_{i}$ and of moment $\mu_{i}$, which can be written

$$
\langle\mathbf{S}\rangle=\sum_{i} \mathbf{S}\left(\mu_{i}\right)\left|\mathbf{r}_{i}\right\rangle\left\langle\mathbf{r}_{i}\right|
$$

The summation can be performed with the following approximation. Because of the significant absorption in the samples, it is meaningful to neglect all interferences effects outside a range of the order of the absorption length of the coherent beam $\ell_{a b s}$. In a medium of size $\ell_{a b s}$, which is assumed to be spherical, interferences can be taken into account exactly in single scattering since $\ell^{*} \gg \ell_{a b s}$. In the basis of Van De Hulst for the polarization, the scattering matrix has the following form

$$
\left\langle S_{i j}\right\rangle \approx n \int_{0}^{\ell_{a b s}} e^{i\left(\mathbf{k}-\mathbf{k}^{\prime}\right) \cdot \mathbf{r}_{i}} S_{i j} d^{3} \mathbf{r}_{i}=f\left(\frac{\ell_{a b s}}{a}\right)^{3} G(v) S_{i j},
$$

where $n$ is the number of particles per unit volume, $G(v)=3(\sin v-v \cos v) / v^{3}$ is the phase function for Rayleigh-Gans scattering and $v=2 k \ell_{a b s} \sin (\theta / 2)$. Relation (10) expresses a well known result in optics: the proportionality of the scattering matrix of a Rayleigh-Gans to the scattering matrix of a Rayleigh scatterer [24]. From the scattering matrix, the scattering cross-section can be computed for the given states of incident and outgoing polarizations. The scattering cross-section, averaged over incident and outgoing polarization, reads:

$$
\left\langle\frac{d \sigma}{d \Omega}\right\rangle=\frac{1}{4} f^{2}\left(\frac{\ell_{a b s}}{a}\right)^{6} G^{2}(v) \sum_{i, j=1,2}\left|S_{i j}\right|^{2} .
$$

To compute the PHE $\eta$ defined in Eq. (1), it is necessary to integrate the scattering cross-section with respect to the direction $\hat{\mathbf{k}}^{\prime}$ including a magneto-transverse projection factor $\hat{\mathbf{B}} \cdot\left(\hat{\mathbf{k}} \times \hat{\mathbf{k}}^{\prime}\right)$. This factor allows to calculate the light flux projected onto the magneto-transverse direction $\hat{\mathbf{B}} \times \hat{\mathbf{k}}$, which is the direction of the normal of the detector in the experiment. After this integration, only terms odd in the magnetic field remain. For unpolarized incident light, the PHE finally reads 


$$
\eta_{\text {unpol }}=\pi \gamma_{1} \frac{\int_{0}^{\pi} d \theta \sin ^{3} \theta \cos \theta G^{2}[v(\theta)]}{\int_{0}^{\pi} d \theta \sin ^{2} \theta\left(1+\cos ^{2} \theta\right) G^{2}[v(\theta)]}
$$

with

$$
\gamma_{1}=\frac{\Im m\left(t_{0} t_{1}^{*}\right)}{\left|t_{0}\right|^{2}}
$$

The integrals in Eq. (12) can be calculated in the limit $k \ell_{a b s} \gg 1$, which gives

$$
\eta_{\text {unpol }}=\frac{3 \gamma_{1}}{2} \frac{\ln \left(k \ell_{a b s}\right)}{k \ell_{a b s}} .
$$

It is interesting to note that when the size parameter of the particles goes to zero $x \rightarrow 0$, the parameters $\gamma_{1}$ and $\eta_{\text {unpol }}$ are independent of the size of the particles. This makes this limit insensitive to polydispersity, which is always difficult to estimate in this kind of experiment.

\section{PHE for polarized incident light}

Polarized incident light may be either circular or linear. A state of linear polarization is described by

$$
E_{1}=\cos \beta, \quad E_{2}=\sin \beta
$$

in Eq. (5b). A sketch of the geometry is shown for this case in Figure 4 . When $\beta=0$, the electric field of the incident light is parallel to the applied magnetic field.

Contrary to the case of unpolarized PHE, several terms present in the scattering matrix now contribute. After having carried out the integration over the outgoing wave vector and the average with respect to the outgoing polarization, the numerator $\Delta I(\mathbf{B})$ and the denominator $I_{0}$ of the PHE take the form

$$
\begin{aligned}
\Delta I(\mathbf{B}) & =\pi A\left[-\frac{1}{2} \operatorname{Im}\left(\mathrm{t}_{2} \mathrm{t}_{1}^{*}\right) \cos (2 \beta)+2 \operatorname{Im}\left(\mathrm{t}_{0} \mathrm{t}_{1}^{*}\right) \sin ^{2} \beta\right] \int_{0}^{\pi} d \theta \sin ^{3} \theta \cos \theta G^{2}[v(\theta)], \\
I_{0} & =2 A\left|t_{0}\right|^{2} \int_{0}^{\pi} d \theta\left[\cos ^{2} \beta+\cos ^{2} \theta \sin ^{2} \beta\right] \sin ^{2} \theta G^{2}[v(\theta)],
\end{aligned}
$$

where $A$ is a known constant of proportionality. In these expressions, the integrals over the scattering angle $\theta$ can be done in the limit $k \ell_{a b s} \gg 1$. For the last equation, this gives

$$
I_{0}=2 A\left|t_{0}\right|^{2} \int_{0}^{\pi} d \theta \sin ^{2} \theta G^{2}[v(\theta)]
$$

which means that the denominator $I_{0}$ of the PHE is independent of the angle $\beta$ characterizing the state of linear incident polarization. The PHE for polarized incident light now reads:

$$
\eta(\beta)=2 \eta_{\text {unpol }}\left[\sin ^{2} \beta-\frac{\gamma_{2}}{4 \gamma_{1}} \cos (2 \beta)\right],
$$

where $\eta_{\text {unpol }}$ is the value of the PHE obtained from Eq. (14) for unpolarized incident light, and $\gamma_{2}$ has been defined in the same way as in Eq. (13) by

$$
\gamma_{2}=\frac{\operatorname{Im}\left(\mathrm{t}_{2} \mathrm{t}_{1}^{*}\right)}{\left|t_{0}\right|^{2}}
$$


Averaging over $\beta$ in Eq. (17) reproduces the value $\eta_{\text {unpol }}$. Two particular cases of Eq. (17) will be of interest: when the linear magnetic birefringence is negligible, one has

$$
\eta(\beta)=2 \eta_{\text {unpol }} \sin ^{2} \beta .
$$

On the other hand, for a dominant contribution of the birefringence, one gets

$$
\eta(\beta)=-\eta_{\text {unpol }} \frac{\gamma_{2}}{2 \gamma_{1}} \cos (2 \beta)=-\frac{3 \gamma_{2}}{4} \frac{\ln \left(k \ell_{a b s}\right)}{k \ell_{a b s}} \cos (2 \beta)
$$

The dependence of the PHE on the state of circular polarization can be done very similarly. A state of circular polarization can be written

$$
E_{1}=\frac{1}{\sqrt{2}}, \quad E_{2}=\frac{ \pm i}{\sqrt{2}}
$$

One finds that the PHE is independent of the state of circular polarization left or right of the incident light

$$
\eta^{ \pm}=\eta_{\text {unpol }}
$$

This value corresponds to the case of linear polarization at the angle $\beta=\pi / 4$, or to the case of unpolarized incident light.

\section{EXPERIMENTAL RESULTS}

\section{A. Dependence of the PHE as function of the field and concentration}

The light intensities $I^{+}$and $I^{-}$, represented in Fig. 1 travel trough optical fibers from the sample to the detectors which are photo-diodes or photo-multipliers. The difference $\Delta I(\mathbf{B})$ is measured using a lock-in. More details on the experimental setup can be found in Ref. 22. The measurement of the phase difference of $\Delta I(\mathbf{B})$ with respect to the magnetic field defines the sign of the PHE. This phase for a true signal should be therefore either 0 or $\pi$ radians. The sign of the PHE is a very important feature which has been seen to be very sensitive to several experimental parameters (Verdet constant, index of refraction, concentration, polarization..).

Our first experimental study addresses the dependence of the PHE as a function of the applied magnetic field. As shown in Fig. 5, the PHE is linear in the applied magnetic field at low field. In ferrofluids, the deviation away from a linear behavior sets in at fields higher than a few hundreds of Oe. Experiments done at higher field show the same saturation which is observed in figure 3 for the Faraday effect. This aggrees with the prediction of Eq. (13) and Eq. (14), and confirms that the PHE is produced by the orientation of the magnetic moments according to the Langevin model. Unless specified otherwise, the rest of the measurements of the PHE was done in the linear regime for the PHE.

The variation of the PHE with volume fraction $f$, is shown in Fig. 1 for a gelled sample, which was prepared with about $1 \%$ of gelatine in volume. The experiment was repeated with liquid samples of ferrofluids. In both cases, a linear behavior with respect to the volume fraction was found. The dispersion of the experimental points in Fig. 6 around the linear behavior is believed to be due to the evaporation of water in the gelled samples, and was absent in the experiment with liquid samples. The approximation used in Eq. (14) is valid since $k \ell_{a b s} \simeq 1.6 \cdot 10^{3}$ for the most concentrated of the samples used of volume fraction $f=0.14 \%$. Since the absorption length $\ell_{a b s}$ is inversely proportional to the volume fraction of the scatterers, Eq. (14) predicts a 
linear dependence of $\eta$ with respect to the volume fraction $f$. This must be caused by a difference $\Delta I(\mathbf{B})$ quadratic in $f^{2}$ while the scattered intensity $I_{0}$ is proportional to $f$. A quadratic behavior for $f^{2}$ in $\Delta I(\mathbf{B})$ means that the PHE involves more than one particle, because the probability of scattering for a photon is proportional to $f$. These experiments confirm our previous statement that a single Rayleigh scatterer is not able to generate the PHE but that more than one Rayleigh scatterers or one scatterer of finite size is necessary to get a non-zero value of the PHE [3].

The PHE per unit magnetic field in the experiment of Fig. 6 is estimated to be $d^{2} \eta / d B d f=3 \cdot 10^{-3} T^{-1}$. In order to compare this value with a theoretical estimate, it is necessary to evaluate the coefficients of the scattering matrix. For a low applied magnetic field, these coefficients can be determined from the anisotropy in the index of refraction [25]. The dielectric constant of the sample is assumed to have the following form at low applied magnetic field:

$$
\epsilon_{i j}=\epsilon_{0} \delta_{i j}+i \epsilon_{F} \epsilon_{i j k} \hat{B}_{k} .
$$

Using our measurements of the Faraday rotation and ellipticity in this sample, we estimate the antisymmetric part of the dielectric constant $\varepsilon_{F}$ to be $d \varepsilon_{F} / d B \simeq(-0.5+$ $1.5 i) \cdot 10^{-4} T^{-1}$. With this value, one can calculate the antisymmetric part of the relative dielectric constant $\kappa_{F}$ of a single particle. The parameter $\gamma_{1}$ can then be obtained from the relation

$$
\gamma_{1}=\frac{\operatorname{Im}\left(\mathrm{t}_{0} \mathrm{t}_{1}^{*}\right)}{\left|t_{0}\right|^{2}}=-3 \operatorname{Im}\left[\frac{\kappa_{\mathrm{F}}}{\left(\kappa_{0}-1\right)\left(\kappa_{0}+2\right)}\right] .
$$

This yields $d^{2} \eta / d B d f \simeq 1,2 \cdot 10^{-3} T^{-1}$, which is to be compared with the slope of Fig. B $d^{2} \eta / d B d f \simeq 3 \cdot 10^{-3} T^{-1}$. Because of experimental uncertainties due to the lack of stability of the gel, this can be considered as a fair agreement. The other experiments with gels which are reported in this paper, were done with sol-gels which are stable.

\section{B. Experimental study of the polarization of the PHE}

The dependence of the PHE on the volume fraction is an important test of the model. However a lot of physical and optical properties depend on the volume fraction, which makes the interpretation of this kind of experiments difficult. The polarization is a much more robust quantity to consider, since it should be independent of many experimental conditions. This section adresses the dependence of the PHE on the incident polarization for liquid and gelled samples of ferrofluids. The polarization of the scattered light is still averaged by the detector, which is not sensitive to the polarization of the light, as was checked experimentally. The opposite experiment, consisting of sending unpolarized light and measuring the polarization of the outcoming light, is equally interesting, but has not been carried out, because it is more difficult to realize.

The first experiment deals with sol-gels of ferrofluids. The gels were produced in the group of J. A. Serughetti from Département de physique des Matériaux de l'Université Claude Bernard in Villeurbanne. The gels were made from the isotropic liquid state with no magnetic field present during the gelation. During the measurement of the PHE, a magnetic field of $250 \mathrm{Oe}$ and of frequency $560 \mathrm{~Hz}$ was applied. In Fig. 月a the normalized PHE is shown as a function of $\beta$, together with the prediction from Eq. (19), when magnetic linear birefringence, the second term in Eq. (17), is assumed to be absent. This assumption is consistant with the observed weak sensitivity of this curve with respect to the amplitude of the applied magnetic field. We have checked that the dependence of the PHE on $\beta$ for linearly polarized incident light 
comes in only from the numerator of $\eta$ in Eq. (1), as no dependence was found in the incoherent background $I_{0}$ as predicted by the model of the previous section. The magnetic birefringence was assumed to be absent in the comparison with our experiments since the it should be inhibited in the gel. Although the general features of the curve are captured by the model, there remains a clear discrepancy between experiments and theory. This discrepancy follows a $\cos \beta^{2} \sin ^{2} \beta$ law, which probably indicates that this point might be explained by taking into account higher scattering orders, such as double scattering for instance.

Figure $7 \mathrm{~b}$ shows measurements of the PHE as function of the angle $\beta$ together with the theoretical prediction of Eq. (20). The sample is a liquid Cobalt ferrofluid, of volume fraction $0.025 \%$, and the applied magnetic field is $40 \mathrm{Oe}$. It can be first noted that the observed dependence of the PHE is completely different from the previous case: the symmetry of the curve with respect to the zero axis is different. There is now a range of values of $\beta$ for which the PHE changes sign, and the effect no longer vanishes at $\beta=0$, but is maximum. Contrary to the case of gelled samples, the experimental result is now compared to the theoretical model for dominant linear magnetic birefringence. In these ferrofluids, the particles are indeed free to reorganize themselves and acquire shape anisotropy, which results in a linear magnetic birefringence generally larger than the Faraday effect. In the same experimental conditions of figure $(\sqrt{b})$ and in a sample length of $3 \mathrm{~mm}$, the Faraday rotation was measured to be $0.1^{\circ}$, to be compared to $1.7^{\circ}$ for the linear magnetic birefringence. The magnetic linear birefringence corresponds to the term $\gamma_{2}$ in Eq. (20), which is an odd function of the magnetic field very much like $\gamma_{1}$ in the unpolarized case. At low magnetic field, $\gamma_{2}$ is proportional to $\mathbf{B}^{3}$, and should therefore generate an oscillation at the modulation frequency of the magnetic field. This time, a very good agreement with the predicted law in $-\cos (2 \beta)$ is found.

The experiment was repeated with circularly polarized incident light, in liquids and in gels, and fully confirmed the result of Eq. (21). There is virtually no measurable difference between $\eta^{+}$et $\eta^{-}$. The value of $\eta^{+}$corresponds indeed to the value of the PHE with linear polarization for an angle $\beta=\pi / 4$.

\section{DYNAMICAL ASPECTS OF THE PHE}

In the last section of our study of the PHE in ferrofluids, some dynamical aspects are discussed, in relation to the rotation of the magnetic moments of the particles induced by the magnetic field in ferrofluids. In section II, it was noted that the main process of relaxation of the magnetic moments in the Cobalt samples is the brownian mechanism. Since the Faraday effect is produced by the orientation of the magnetic moments, it should follow the same relaxation law as the magnetization. This relaxation of the Faraday rotation as a function of the frequency of the magnetic field was indeed observed [27]. The characteristic relaxation time can be obtained either from the study of the frequency dependence of the Faraday rotation or from the study of the transient response as the magnetic field is turned off. Both methods provide comparable values of the relaxation time. From Eq. (1), it can be noted that the relaxation time for brownian relaxation is directly linked with the viscosity of the suspension. Using this idea, Bacri et al. in Paris and B. Payet in St-Etienne have experimentally shown that measurements of relaxation times could be used to determine the viscosity of the carrier liquid $\nu$. A viscosimeter based on this principle could achieve an accuracy of a few percents and a range of several decades of viscosity. More recently, measurements of relaxation times are now used for biological applications of ferrofluids [28].

The magnetic susceptibility of ferrofluids is well described by Debye theory, originally applied to dielectrics but also very useful for magnetic liquids. Debye theory 
essentially relies on the following hypotheses:

1. All magnetic moments of the particles are fixed with respect to the particles

2. All particles have identical dimensions and magnetic moments

3. There is no interaction between the particles.

Within Debye's model, the magnetic susceptibility is

$$
\chi\left(\omega_{H}\right)=\frac{\chi_{0}}{1+i \omega_{H} \tau}=\left|\chi\left(\omega_{H} \tau\right)\right| e^{i \varphi\left(\omega_{H} \tau\right)},
$$

where $\omega_{H}$ stands for the frequency of the magnetic field and $\tau$ for the relaxation time of the liquid. The proportionality of the magnetic susceptibility (23) and the Faraday rotation (measured both in amplitude and phase) has been established in Ref. [27. Therefore, it is expected that the PHE should be also proportional to this complex magnetic susceptibility. It is very important not to confuse the phase $\varphi\left(\omega_{H} \tau\right)$ defined in Eq. 23) with the phase of the scattered light, which is often written as a complexvalued quantity. The phase $\varphi\left(\omega_{H} \tau\right)$ is defined unambiguously with respect to the magnetic field and varies at the frequency of the magnetic field $\omega_{H}$, whereas the phase of the scattered light oscillates at optical frequency.

In order to show the applicability of Debye's theory to the PHE in ferrofluids, the amplitude and phase of the PHE were measured as function of the product $\omega_{H} \tau$, as shown in Fig. 8. Experiments were carried out in samples of different viscosities by diluting a suspension of (liquid) ferrofluid with water, which was originally a solution in glycerol. Three samples of identical volume fraction $f=0.025 \%$ in ferrofluid were used: the original solution with $100 \%$ of glycerol of viscosity $1093 \mathrm{~Pa} . \mathrm{s}$, a solution with $90 \%$ of glycerol of viscosity $384 \mathrm{~Pa} . \mathrm{s}$, and a solution with $75 \%$ of glycerol of viscosity $60 \mathrm{~Pa} . \mathrm{s}$ at $25^{\circ}$. A magnetic field of $100 \mathrm{Oe}$ was applied during the experiment. The curves for different viscosities do superimpose on each other, which confirms that the PHE only depends on the product $\omega_{H} \tau$.

The saturation of the PHE as function of the magnetic field and the measurement of relaxation time both confirm that the origin of the PHE resides in the orientation of the magnetic moments of the particles, just like for the Faraday effect. The observed behavior as function of frequency in figure 8 is compared with the prediction of the Debye theory of Eq. (23). The measurements of the amplitude and phase of the PHE agrees with the Debye model when the parameter $\omega_{B} \nu$ is below a value of about $10^{5} \mathrm{~Pa}$. Above this value, a deviation is seen between the experiment and the theoretical curve of the amplitude of the PHE, and a more dramatic deviation is seen in the phase at high frequency. The experimental setup should not be responsible for this deviation, even at the highest frequency of $\omega_{B}=1.875 \mathrm{kHz}$. The PHE at this frequency is about two orders of magnitude smaller as compared to its value at low frequency, but the noise level is still very low for these measurements (less than 1\%). In these rather dilute samples, the origin of this deviation, the caracteristic time of which is about $8 \mathrm{~ms}$, is unclear.

\section{CONCLUSION}

We have presented a theoretical model of the PHE, which captures the essential features of the experiments reported in this paper. This work confirms our understanding of the PHE in a system of Rayleigh absorbing scatterers, and its connection with the Faraday effect. In ferrofluids, as shown by static and dynamic measurements, the Faraday effect and the PHE were both found to be produced by the orientation of the magnetic moments. This work on the PHE in ferrofluids could also be useful to improve our understanding of complex media containing ferrofluid particles, such 
as media in which the particles are not free or are only partially free to move. The polarization dependence of the PHE was found to be sensitive to the degrees of freedom of the particles in the medium. In the magneto-transverse geometry, this type of experiment could provide information complementary to the one provided by the magnetic linear birefringence.

We wish to thank H. Roux, C. Bovier and G. Rikken for their help in the experimental part, J. Ferré, R. Perzynsky, and J-C. Bacri for the enlightening discussions on ferrofluids, and T. Lubensky for stimulating discussions.

[1] B. A. Van Tiggelen. Transverse Diffusion of light in Faraday-active media. Phys. Rev. Lett., 75(3):42224, 1995.

[2] G. L. J. A. Rikken and B. A. Van Tiggelen. Observation of magnetically induced transverse diffusion of light. Nature, 381:54-55, 1996.

[3] D. Lacoste, B. A. Van Tiggelen, G. L. J. A. Rikken, and A. Sparenberg. Optics of a Faraday-active Mie sphere. J. Opt. Soc. Am. A, 15:1636-1642, 1998.

[4] D. Lacoste and B. A. Van Tiggelen. Transport mean free path for magneto-transverse light diffusion. Europhys. Lett., 45(6):721-725, 1999.

[5] S. Wiebel, A. Sparenberg, G. L. J. A. Rikken, D. Lacoste, and B. A. Van Tiggelen. The photonic Hall effect in absorbing media. Submitted, 2000.

[6] P. N. Argyres. Theory of the Faraday and Kerr effects in ferromagnetics. Phys. Rev., 97:334-345, 1955.

[7] G. S. Krinchik. Ferromagnetic Hall Effect at optical frequencies and inner effective magnetic field of ferromagnetic metals. J. Appl. Phys., 35(3):1089-1092, 1964.

[8] R. E. Rosensweig. Ferrohydrodynamics. Dover, New York, 1997.

[9] P. G. de Gennes and P. A. Pincus. Pair Correlations in a ferromagnetic colloid. Phys. Kondens. Materie, 11:188-198, 1970.

[10] M. J. Stevens and G. S. Grest. Coexistence in dipolar fluids in a field. Phys. Rev. Lett., 72(23):3686-9, 1994.

[11] S. Neveu., F. A. Tourinho, J.-C. Bacri, and R. Perzynski. Magnetic birefringence of Cobalt ferrite ferrofluids. Colloids Surface A: Physicochem. Eng. Aspect, 80:1-10, 1993.

[12] G. S. Krinchik, K. M. Mukimov, Sh. M. Sharipov, A. P. Khrebtov, and E. M. Speranskaya. The permittivity tensor and increase in the transmittance of the spinel ferrites upon their conversion into single-sublattice structures. Sov. Phys. JETP, 49(6):1074-1079, 1979.

[13] J. W. D. Martens and W. L. Peeters. An ellipsometric and magneto-optical study of cobalt ferrite single crystals. Solid State Comm., 41(9):667-669, 1982.

[14] C. F. Bohren and D. R. Huffman. Absorption and Scattering of Light by Small Particles. Wiley, New York, 1983.

[15] H. W. Davies and J. P. Llewellyn. Magneto-optical effects in ferrofluids. J. Phys. D., Appl. Phys., 13:2327-2336, 1980.

[16] N. Yusuf, A. Roussan, and H. M. El-Ghanem. Determination of Faraday rotation in a ferrofluid. J. Magn. Magn. Mat., 65:282-4, 1987.

[17] D. Jamon, F. Donatini, J. Monin, M. Rasa, V. Socoliuc, O. Filip, D. Bica, and V. Sofonea. Concentration dependence of magnetization and magneto-optical effects in a ferrofluid with double layer stabilized particles. J. Magn. Magn. Mat., 201:174-7, 1999.

[18] P. Daveze. Contribution à l'étude de propriétés magnéto-optiques des liquides magnétiques. Applications opto-électroniques. PhD thesis, Université Jean Monnet à St-Etienne, 1996.

[19] E. Hasmonay, E. Dubois, J-C. Bacri, R. Perzynsky, Y. L. Raikher, and V. I. Stepanov. Static magnetooptical birefringence of size-sorted $\gamma-\mathrm{Fe}_{2} \mathrm{O}_{3}$ nanoparticles. Euro. Phys. J. B, 5(4):859-67, 1998.

[20] J-C. Bacri and D. Gorse. Critical behaviour of the elastic constant and the friction coefficient in the gel 
phase of gelatin. J. Phys., 44:985-991, 1983.

[21] F. Bentivegna, M. Nyvlt, J. Ferré, J.P Jamet, A. Brun, S̆. Višn̆ovský, and R. Urban. Magnetically textured $\gamma-\mathrm{Fe}_{2} \mathrm{O}_{3}$ nanoparticles in a silica gel matrix: Optical and magneto-optical properties. J. Appli. Phys., 85(4):2270-8, 1999.

[22] S̆. Višn̆ovský. Optics of magnetic multilayers. Czech. J. Phys., 41(2):663-27, 1991.

[23] F. Donatini, D. Jamon, J. Monin, and S. Neveu. Experimental investigation of longitudinal magnetooptic effects in four ferrite ferrofluids in visible-near infrared spectrum. IEEE Trans. Magn., 35(5):43114317, 1999.

[24] H. C. Van de Hulst. Light Scattering by Small Particles. Dover, New York, 1980.

[25] B. A. Van Tiggelen, R. Maynard, and T. M. Nieuwenhuizen. Theory for multiple light scattering from Rayleigh scatterers in magnetic fields. Phys. Rev. E, 53(3):2881-2908, 1996.

[26] J. J. M. Janssen and J. A. A. J. Perenboom. Magneto-optical phenomena in magnetic fluids: the influence of orientation of anisotropic scatterers. J. Magn. Magn. Mat., 81:14-24, 1989.

[27] B. Payet, F. Donatini, and G. Noyel. Longitudinal magneto-optical study of Brown relaxation in ferrofluids and transient methods. Applications. J. Magn. Magn. Mat., 201:207-210, 1999.

[28] R. Kötitz. Private communication. 2000. 


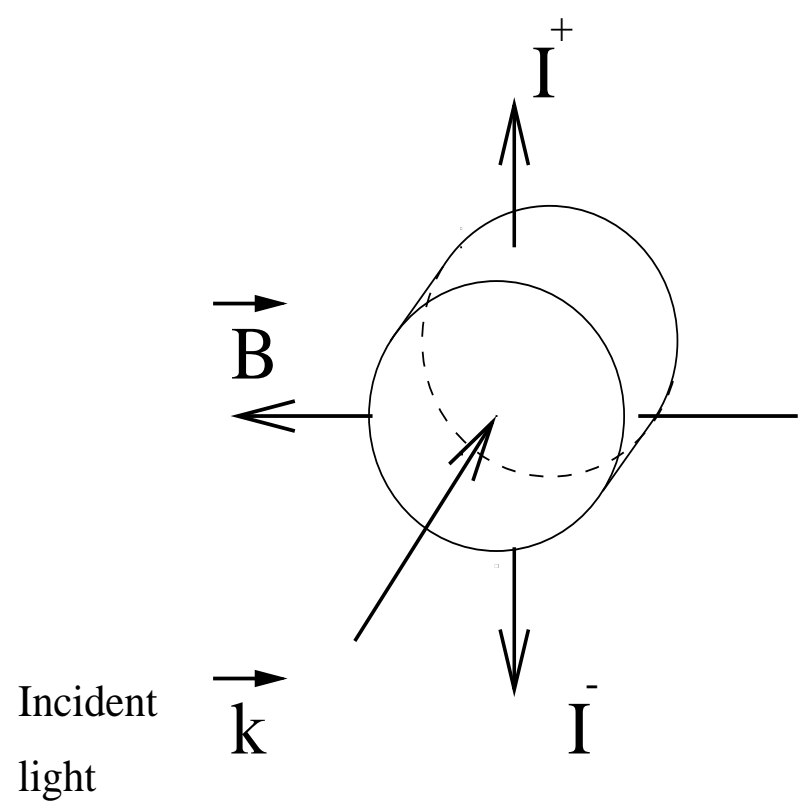

FIG. 1. Sketch of the geometry of the PHE. The sample is shown as a cylinder. The scattered intensities above (and below) the plane containing the incident light direction and the magnetic field are called respectively $I^{+}$and $I^{-}$. The difference $\Delta I(\mathbf{B})$ is the photonic equivalent of a difference of potential in the electronic Hall effect.

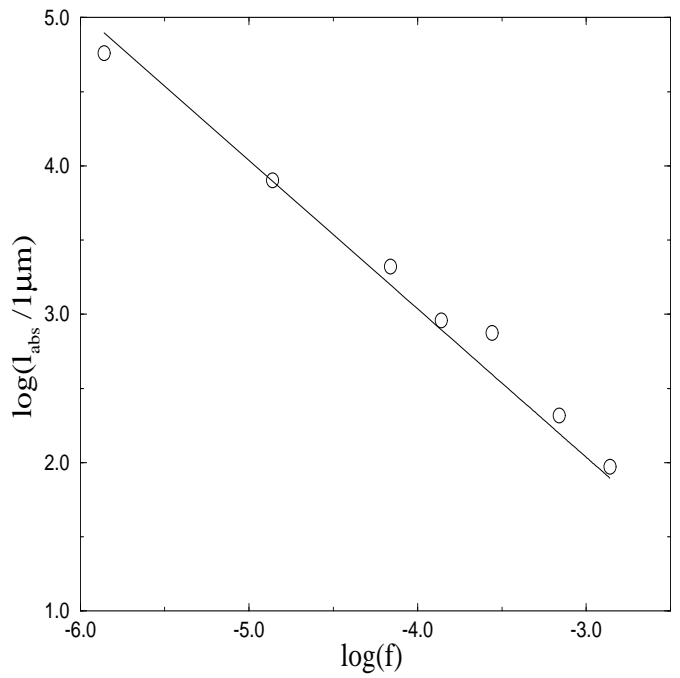

FIG. 2. Logarithm of the absorption length $\log _{10}\left(\ell_{a b s} / 1 \mu \mathrm{m}\right)$ as function of the logarithm of the volume fraction $f$ at the wavelength $\lambda_{0}=477 \mathrm{~nm}$, for several aqueous solutions of $\mathrm{Fe}_{2} \mathrm{CoO}_{4}$. Points represent measurements and the line results from Lambert-Beer's law (the slope is estimated from Rayleigh scattering theory for a monodisperse distribution of particles of radius $a=6 \mathrm{~nm}$ using the index of refraction of the material at this wavelength $2.6-0.7 i$.) 


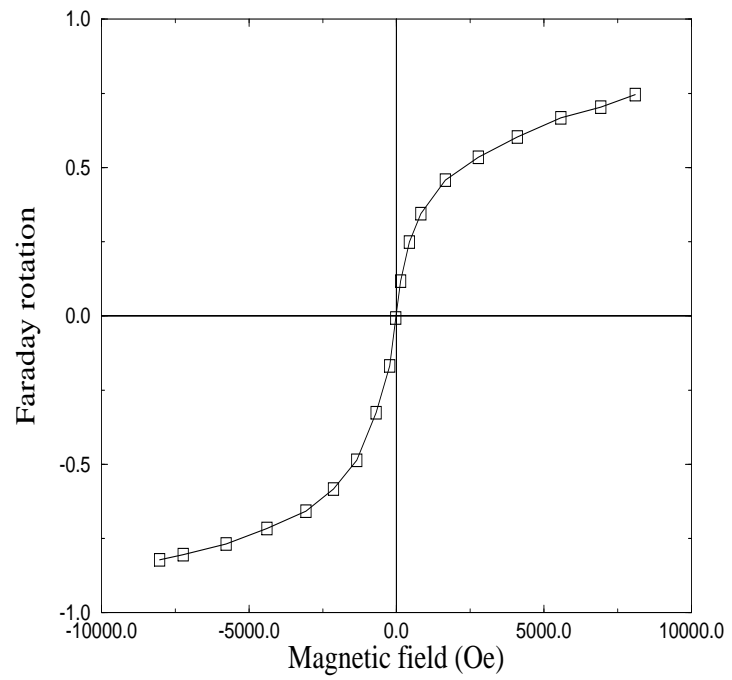

FIG. 3. Normalized measurements of Faraday rotation and ellipticity in a sol-gel of Cobalt ferrite of volume fraction $0.04 \%$. The superposition of the normalized curves for the two effects is an indication of their common origin, both effects scaling with the Langevin function introduced in section II. For the maximum field $8224 \mathrm{Oe}$, the Faraday rotation is $0.7^{\circ}$ and the ellipticity $1.91^{\circ}$ in a sample holder of length $2 \mathrm{~mm}$. The wavelength in this experiment was $\lambda_{0}=633 \mathrm{~nm}$, and these measurements were taken in a static magnetic field.
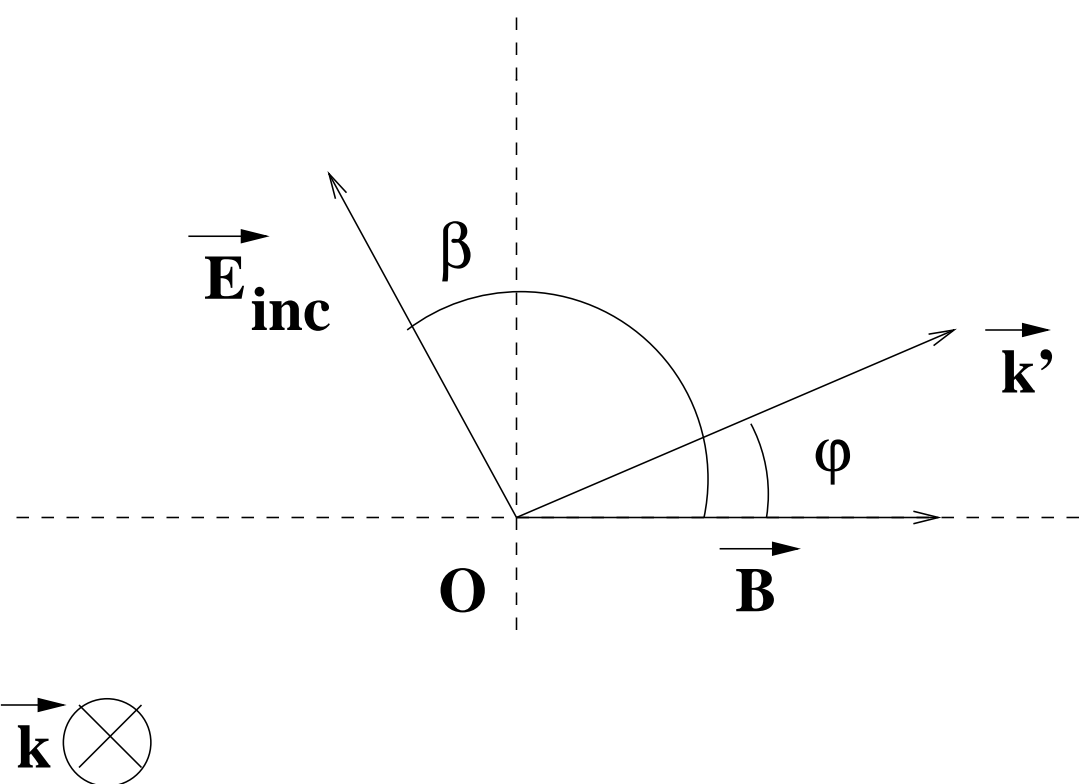

FIG. 4. Sketch of the geometry in the frame of the laboratory. The vector $\mathbf{B}$ defines the axis $x$, the magneto-transverse direction is along the axis $y$, and the incident wave vector is along $z$. The angle $\beta$ characterizes the state of linear polarization of the incident electric field. 

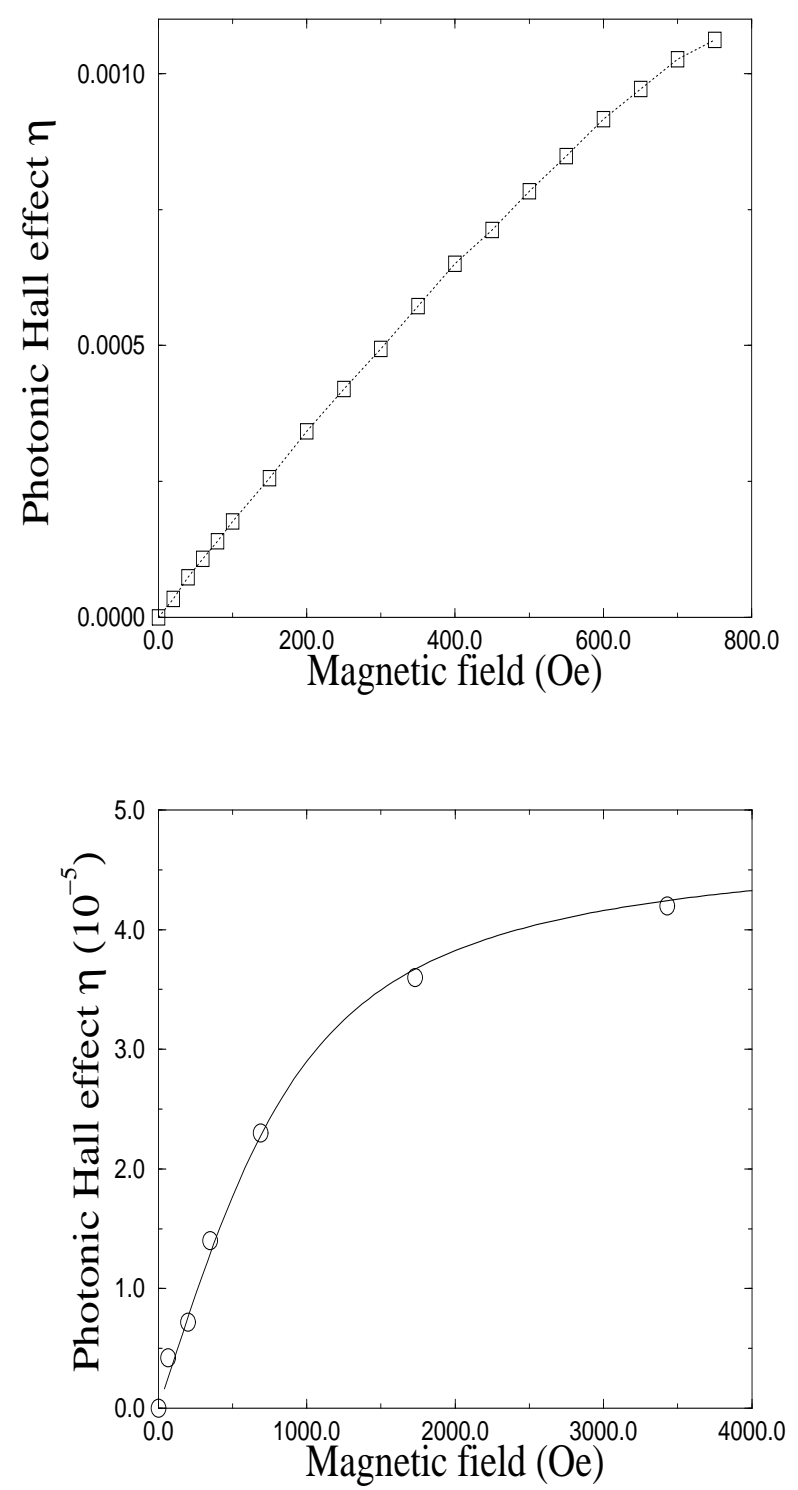

FIG. 5. (a) The squares represent measurements of the PHE $\eta$ at low field, as function of the applied magnetic field. The curve only connects the experimental points. It can be noted that the PHE is linear in magnetic fields smaller than about a hundred of Oe. This experiment was done with the same sample than in Fig. (3). (b) Same experiment with similar samples but with higher magnetic field, so that the saturation of the PHE is now clearly visible. The circles are experimental points and the line is a fit using the Langevin function. 


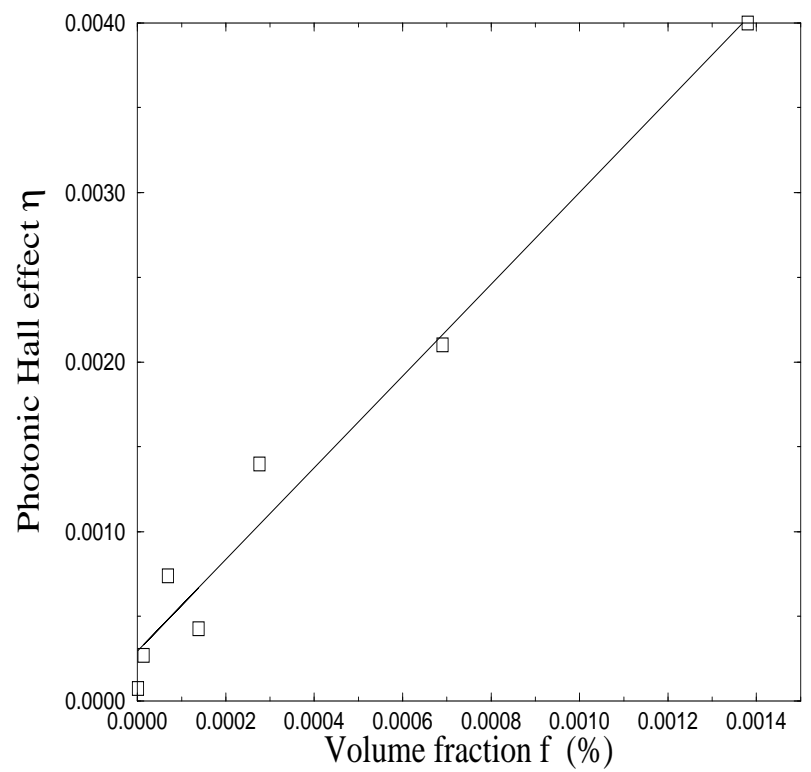

FIG. 6. Points are experimental measurements of PHE $\eta$ as function of the volume fraction $f$. Samples are gels made with $1 \%$ of gelatine in volume and Cobalt ferrofluid. A linear fit gives the slope per unit of magnetic field $d^{2} \eta / d B d f=3 \cdot 10^{-3} T^{-1}$.

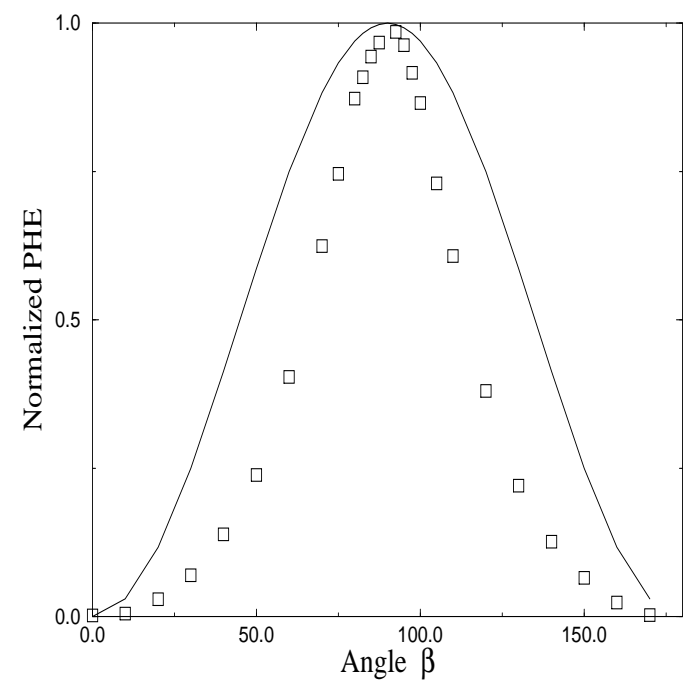




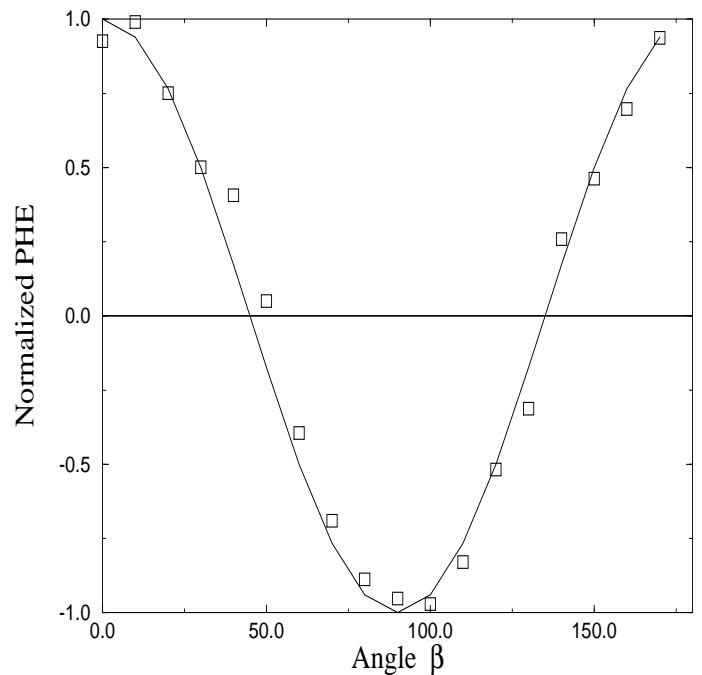

FIG. 7. (a) Normalized PHE for a sol-gel sample of ferrofluid as function of the angle $\beta$ of the incident linear polarization. Points are experimental and the curve is a prediction from Eq. (19). A magnetic field of $250 \mathrm{Oe}$ and frequency $560 \mathrm{~Hz}$ was applied on the same sample studied in Fig. $3 \mathrm{~b}$. (b) Normalized PHE for liquid ferrofluid as function of $\beta$, together with the prediction of Eq. (20). A magnetic field of 40Oe and frequency $20 \mathrm{~Hz}$, was applied on a sample of volume fraction $0.025 \%$ of aqueous Cobalt ferrite ferrofluid.

(a)

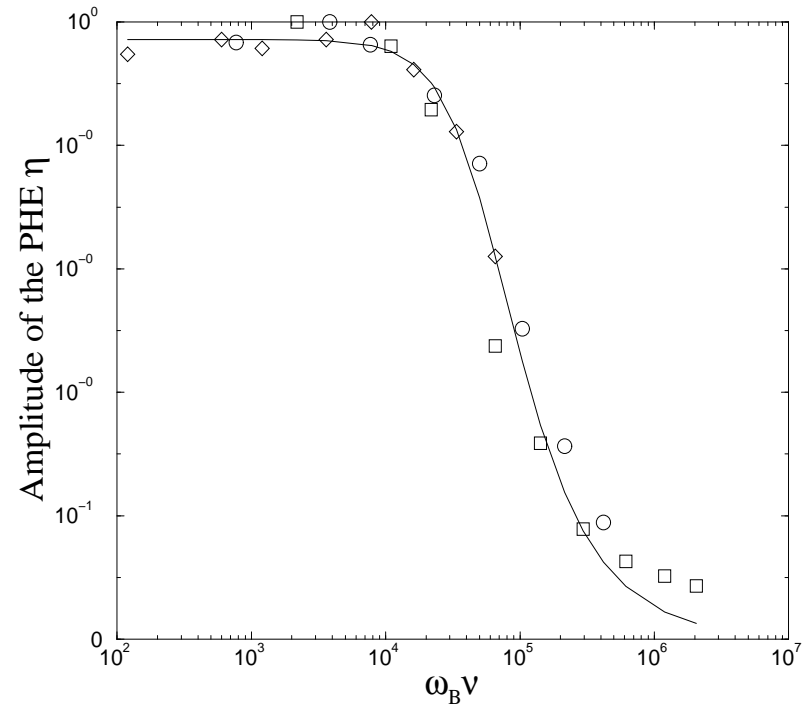


(b)

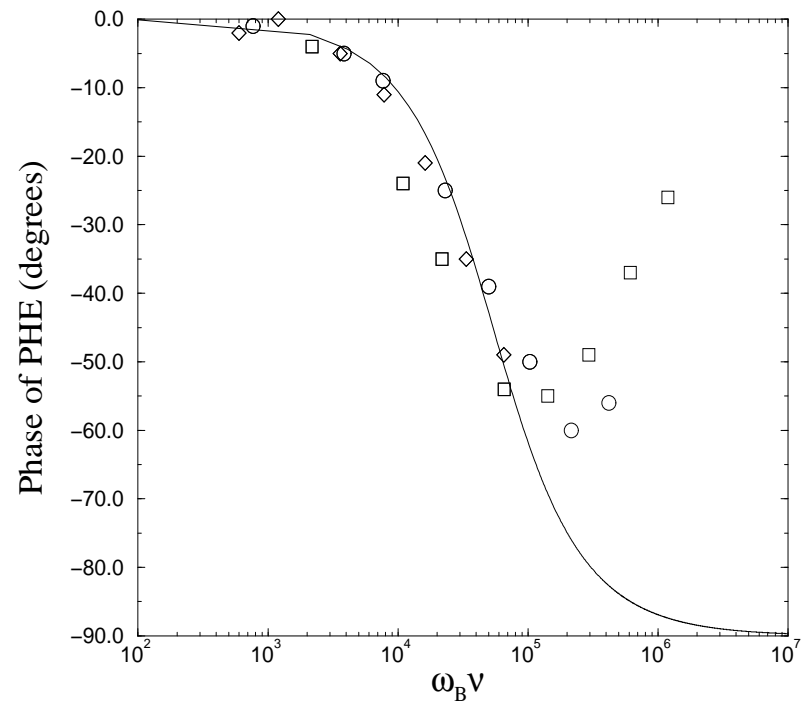

FIG. 8. Amplitude (a) and phase (b) of normalized PHE as a function of the product of the frequency of the magnetic field $\omega_{B}$ and the viscosity of the suspension $\nu$ for samples of different viscosity: the original solution with $100 \%$ de glycerol (squares), a solution with $90 \%$ of glycerol (circles), and a solution with $75 \%$ of glycerol (diamonds). The solid curves in (a) and (b) are fits using Eq. (23). The experiment has been realized with a field of $100 \mathrm{Oe}$ and with samples of volume fraction $0.025 \%$. The Debye model predicts a behavior of the phase at high frequency, which is not seen in the experiment. 